\title{
Padma 28 bei Zytostatika-induzierten Dysästhesien und Ödemen der Hände - ein Fall aus der Praxis
}

\author{
Bettina Kneip \\ Allgemeine Innere Medizin FMH, Institut für Naturheilkunde, UniversitätsSpital Zürich, Schweiz
}

\author{
Schlüsselwörter \\ Dysästhesien · Ödeme · Zytostatika · Padma 28 . \\ Mikrozirkulation
}

\section{Zusammenfassung}

Hintergrund: Die pflanzliche Komplexformel Padma 28 wird vor allem bei durchblutungsbedingten Parästhesien, Schwere- und Spannungsgefühl in den Beinen und Armen und beim Einschlafen von Händen und Füßen eingesetzt. Fallbericht: Im vorgestellten Fall einer Patientin mit Mammakarzinom, Status nach vollständiger Mastektomie, traten starke, Zytostatika-induzierte Dysästhesien und Ödeme beider Hände auf. Diese machten einfache, tägliche Verrichtungen unmöglich. Eine Therapie mit hochdosiertem Vitamin B6 blieb erfolglos. Mit dem Ziel, die Mikrozirkulation zu verbessern und so die empfindlichen Nervenendigungen zu schützen, wurde Padma 28 eingesetzt. Diese Behandlung zeigte trotz fortdauernder Chemotherapie bereits nach 2 Wochen eine Verbesserung der Dysästhesien; nach 5 Wochen waren die Empfindungsstörungen und Ödeme der Hände völlig verschwunden. Schlussfolgerung: Padma 28 scheint somit geeignet zur adjuvanten Anwendung bei Parästhesien und Schwellungen, die im Rahmen einer Chemotherapie durch Zytostatika hervorgerufen wurden.

\section{Einleitung}

Die häufigsten Nebenwirkungen von Zytostatika betreffen unter anderem Störungen und Schäden an Haut und Schleimhäuten sowie Blutbildungsstörungen. Die Folgen sind für Tumorpatienten, zusätzlich zur Grunderkrankung,

Hinweis: Padma 28 (Swissmedic No. 58436) ist in der Schweiz auch unter den Namen Padmed Circosan (Swissmedic No. 60131) und Arteria-vita (Swissmedic No. 62863) erhältlich und kassenzulässig.

\author{
Keywords \\ Dysesthesia - Edema - Cytotoxic drugs - Padma 28 . \\ Microcirculation
}

\section{Summary}

Padma 28 in Cytostatic-Induced Dysesthesia and Edema of the Hands - a Case from Practice

Background: The herbal complex formula Padma 28 is mostly used in circulation-induced paresthesia, in feelings of heaviness and tension in the legs and arms, and in numbness of the hands and feet. Case Report: In the case presented here of a patient with breast cancer, status after full mastectomy, severe cytostatic-induced edema, and dysesthesia occurred on both hands, which made simple, daily tasks impossible. Treatment with high doses of vitamin B6 was unsuccessful. Padma 28 was given under the rationale to improve the microcirculation and thus to protect the delicate nerve endings. Despite ongoing chemotherapy, this treatment led to an improvement of the dysesthesia after 2 weeks, and after 5 weeks, the sensory disturbances and edema of the hands had completely disappeared. Conclusion: Padma 28 seems therefore suitable for adjuvant use in paresthesia and swelling caused by cytotoxic drugs during chemotherapy.

\begin{tabular}{ll}
\hline KARGER & @ 2013 S. Karger GmbH, Freiburg \\
1661-4119/13/0208-0033\$38.00/0 \\
$\begin{array}{l}\text { Fax +49 7614520714 } \\
\text { Information@Karger.com } \\
\text { www.karger.com }\end{array}$ & $\begin{array}{l}\text { Accessible online at: } \\
\text { www.karger.com/fok }\end{array}$
\end{tabular}

Dr. med. Bettina Kneip

Allgemeine Innere Medizin FMH

Institut für Naturheilkunde

UniversitätsSpital Zürich

Rämistrasse 100, Postfach 131

8091 Zürich, Schweiz 


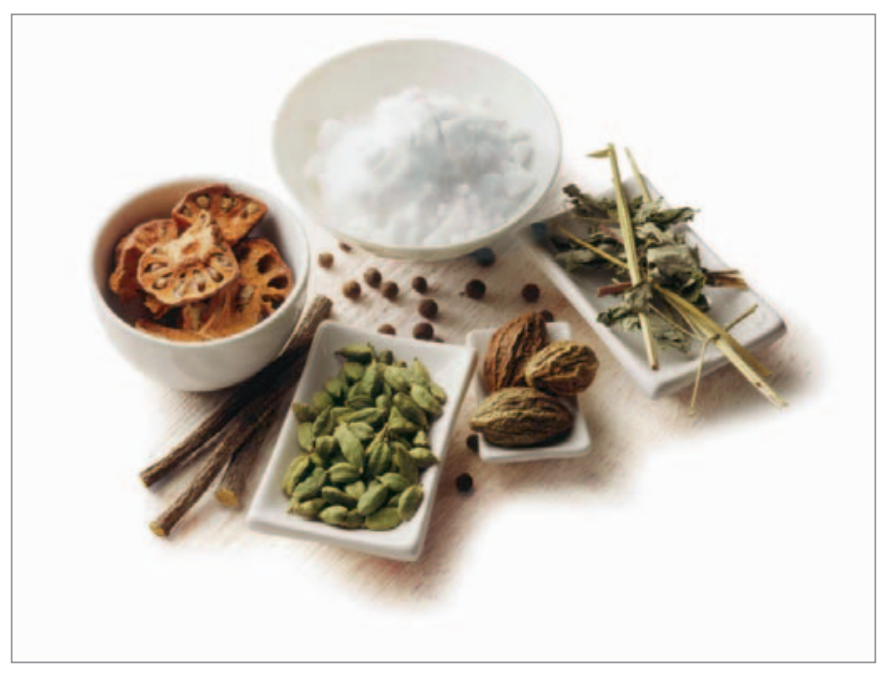

Abb. 1. Einige der pflanzlichen Inhaltsstoffe der Komplexformel Padma 28.

sien wie Kribbeln, Ameisenlaufen, Schwere- und Spannungsgefühl in den Beinen und Armen, Einschlafen von Händen und Füßen angewendet, die durch arterielle Durchblutungsstörungen, aber auch durch Störungen der Mikrozirkulation verursacht werden können. Im hier beschriebenen Fall wurde das Präparat aufgrund von Zytostatika-induzierten Dysästhesien und Ödemen eingesetzt.

\section{Fallbericht}

\section{Patientin}

Die Patientin zeigte folgende Charakteristika: 46 Jahre, mit multifokalem, invasiv-duktalem Mammakarzinom links pT2 (m), pN1a (1/43), MO, G3, L1, VO, Östrogenrezeptor (ER)-negativ, Progesteronrezeptor (PR)negativ, HER2neu-negativ, Proliferationsfraktion hoch. Nebendiagnosen sind: Asthma bronchiale, zurzeit ohne medikamentöse Behandlung, und Morbus Crohn, wobei die Patientin unter $2 \times 50 \mathrm{mg}$ Azathioprin beschwerdefrei ist. Es besteht ein Status nach 3 Spontangeburten (1982, 1983, 1986). In der Familienanamnese väterlicherseits war die Großmutter an einem Ovarialkarzinom erkrankt.

Anfang November 2011 erfolgte eine vollständige Mastektomie mit Sentinellymphnodektomie und Axilladissektion, eine LevonogestrelSpirale wurde entfernt. Es wurde mit einer adjuvanten Chemotherapie begonnen und die Patientin erhielt 3 Zyklen FEC (Fluoruracil $500 \mathrm{mg} / \mathrm{m}^{2}$, Epirubicin $100 \mathrm{mg} / \mathrm{m}^{2}$, Cyclophosphamid $500 \mathrm{mg} / \mathrm{m}^{2}$ ) und 3 Zyklen Docetaxel $\left(100 \mathrm{mg} / \mathrm{m}^{2}\right)$. Eine endokrine Therapie war bei negativem Hormonrezeptorstatus nicht indiziert, eine Radiotherapie wurde bei Status nach vollständiger Mastektomie nicht durchgeführt.
Einige Tage nach Beginn der Zytostatikatherapie klagte die Patientin über zunehmende Dysästhesien an beiden Händen, vor allem an den Fingerkuppen. Trotz Behandlung mit Vitamin B6 oral hochdosiert $(3 \times$ $150 \mathrm{mg}$ für etwa 6 Wochen) nahmen die Beschwerden zu und es bildeten sich Ödeme an beiden Händen, sodass tägliche Verrichtungen wie z.B. Flaschen öffnen, Gemüse schneiden oder die Bluse zuknöpfen unmöglich wurden.

\section{Therapie}

Wir interpretierten die neurosensorischen Störungen als Reaktion auf Docetaxel. Da die Patientin bereits von der Onkologie mit Vitaminen der B-Klasse versorgt worden war, entschieden wir uns zur Unterstützung der Nutrition der vulnerablen Nervenendigungen im Rahmen der Verbesserung der Mikrozirkulation für eine Behandlung mit Padma 28, 3 × 2 Kapseln/Tag.

\section{Ergebnis}

Bereits nach 2 Wochen gingen die Dysästhesien trotz Fortführung der belastenden Chemotherapie deutlich zurück, nach 5 Wochen war die Patientin vollkommen frei von den Empfindungsstörungen und Ödemen beider Hände.

\section{Diskussion und Schlussfolgerung}

Der vorliegende Fallbericht zeigt, dass beim Auftreten von Empfindungsstörungen unter Chemotherapie eine additive Behandlung mit Padma 28 versucht werden kann, dies im Hinblick auf eine Verbesserung der Mikrozirkulation und zum Schutz der empfindlichen Nervenendigungen. Bekannt ist, dass wohl nicht nur die arterielle Durchblutung verbessert wird, wie Studien zu Padma 28 bei peripherer arterieller Verschlusskrankheit (PAVK) zeigen [4]. Wie Beobachtungen zeigten [5], kann Padma 28 auch einen günstigen Einfluss auf den venösen Rückfluss haben, was zu einem schnelleren Abtransport von lokaltoxischen Substanzen führt und somit den Flurschaden zu minimieren scheint. Außerdem ist das Tibetische Vielstoffgemisch in der Lage, im Gewebe antioxidativ zu wirken und somit die Anzahl der freien Radikale, die bei einer Chemotherapie unweigerlich entstehen, zu reduzieren $[6,7]$. Die Verbesserung der Mikrozirkulation durch Padma 28 scheint außerdem eine bessere Versorgung mit Mikronährstoffen zu gewährleisten, was in der Folge für die geschädigten Neuronen offenbar eine nicht unerhebliche Unterstützung in der Regenerationsphase darstellt.

\section{Disclosure Statement}

Es bestehen keine Interessenskonflikte.

\section{Literatur}

1 Fachinformation auf www.kompendium.ch (Information vom 18.02.2013).

$\checkmark 2$ Argyriou AA, Koltzenburg M, Polychronopoulos P, Papapetropoulos S, Kalofonos HP: Peripheral nerve damage associated with administration of taxanes in patients with cancer. Crit Rev Oncol Hematol 2008;66:218-228.

3 Melzer J, Saller R: Das tibetische Kombinationspräparat Padma 28 bei peripherer arterieller Verschlusskrankheit. Internist Prax 2010;2:403-407.
4 Melzer J, Brignoli R, Diehm C, Reichling J, Do DD, Saller R: Treating intermittent claudication with Tibetan medicine Padma 28: does it work? Atherosclerosis 2006;189:39-46.

5 Locher A: Padma 28 bei venösen Beinbeschwerden - ein Fallbericht. Schweiz Z Ganzheitsmed 2012;24: 272-273.
6 Suter M, Richter C: Anti- and pro-oxidative properties of PADMA 28, a Tibetan herbal formula. Redox Rep 2000;5:17-22.

7 Brunner-La Rocca HP, Schindler R, Schlumpf M, Saller R, Suter M: Effects of the Tibetan herbal preparation Padma 28 on blood lipids and lipid oxidisability in subjects with mild hypercholesterolaemia. Vasa 2005;34:11-17. 